\title{
Privacy preserving data anonymization of spontaneous ADE reporting system dataset
}

Wen-Yang Lin ${ }^{*}$, Duen-Chuan Yang ${ }^{\dagger}$ and Jie-Teng Wang

From The ACM Ninth International Workshop on Data and Text Mining in Biomedical Informatics

Melbourne, Australia. 23 October 2015

\begin{abstract}
Background: To facilitate long-term safety surveillance of marketing drugs, many spontaneously reporting systems (SRSs) of ADR events have been established world-wide. Since the data collected by SRSs contain sensitive personal health information that should be protected to prevent the identification of individuals, it procures the issue of privacy preserving data publishing (PPDP), that is, how to sanitize (anonymize) raw data before publishing. Although much work has been done on PPDP, very few studies have focused on protecting privacy of SRS data and none of the anonymization methods is favorable for SRS datasets, due to which contain some characteristics such as rare events, multiple individual records, and multi-valued sensitive attributes.
\end{abstract}

Methods: We propose a new privacy model called MS $\left(k, \theta^{*}\right)$-bounding for protecting published spontaneous ADE reporting data from privacy attacks. Our model has the flexibility of varying privacy thresholds, i.e., $\theta^{*}$, for different sensitive values and takes the characteristics of SRS data into consideration. We also propose an anonymization algorithm for sanitizing the raw data to meet the requirements specified through the proposed model. Our algorithm adopts a greedy-based clustering strategy to group the records into clusters, conforming to an innovative anonymization metric aiming to minimize the privacy risk as well as maintain the data utility for ADR detection. Empirical study was conducted using FAERS dataset from 2004Q1 to 2011Q4. We compared our model with four prevailing methods, including k-anonymity, $(X, Y)$-anonymity, Multi-sensitive $/$-diversity, and $(a, k)$ -anonymity, evaluated via two measures, Danger Ratio $(D R)$ and Information Loss (IL), and considered three different scenarios of threshold setting for $\theta^{*}$, including uniform setting, level-wise setting and frequency-based setting. We also conducted experiments to inspect the impact of anonymized data on the strengths of discovered ADR signals.

Results: With all three different threshold settings for sensitive value, our method can successively prevent the disclosure of sensitive values (nearly all observed DRs are zeros) without sacrificing too much of data utility. With non-uniform threshold setting, level-wise or frequency-based, our MS $\left(k, \theta^{*}\right)$-bounding exhibits the best data utility and the least privacy risk among all the models. The experiments conducted on selected ADR signals from MedWatch show that only very small difference on signal strength (PRR or ROR) were observed. The results show that our method can effectively prevent the disclosure of patient sensitive information without sacrificing data utility for ADR signal detection.

(Continued on next page)

\footnotetext{
* Correspondence: wylin@nuk.edu.tw

${ }^{\dagger}$ Equal contributors

Department of Computer Science and Information Engineering, National

University of Kaohsiung, Nanzih District, Kaohsiung 811, Taiwan, R.O.C
} 
(Continued from previous page)

Conclusions: We propose a new privacy model for protecting SRS data that possess some characteristics overlooked by contemporary models and an anonymization algorithm to sanitize SRS data in accordance with the proposed model. Empirical evaluation on the real SRS dataset, i.e., FAERS, shows that our method can effectively solve the privacy problem in SRS data without influencing the ADR signal strength.

Keywords: Adverse drug reaction, ADR signal detection, Data anonymization, Privacy preserving data publishing, Spontaneous reporting system

\section{Background}

It is well known that a new drug before hitting the market needs to undergo a series of clinical trials to reveal all possible adverse drug reactions (ADRs). Unfortunately, many serious ADRs cannot be disclosed in the premarketing stage through the limited number of volunteers participate in clinical trials; on the contrary, they can only be identified through long term surveillance of extensive usages of the drug on the masses. Therefore, most of highly developed countries have established various spontaneous reporting systems (SRSs) to collect adverse drug events (ADEs) as a data repository for ADR detection and analysis, e.g., the FDA Adverse Event Reporting System (FAERS) of the US Food and Drug Administration (FDA) [1], the UK Yellow Card scheme [2], and the MedEffect Canada [3], among others.

Usually, the data collected by the SRSs contain sensitive personal health information that should be protected to prevent the identification of individuals. This procures the need of anonymizing the raw data before being published, namely privacy-preserving data publishing (PPDP) [4]. Although in the past few years there have been a lot of researches on this topic, none of the anonymization methods is favorable for SRS datasets, due to which contain some characteristics, including rare events, multiple individual records, and multi-valued sensitive attributes.

In this paper, we present a new privacy-preserving model, called $\operatorname{MS}(k, \theta)$-bounding, for protecting the published spontaneous ADE reporting data from privacy attacks. We also propose an anonymization algorithm for sanitizing the raw data to meet the requirements specified through the proposed model. Empirical study conducted using FAERS datasets show that our method can effectively prevent the disclosure of patient sensitive information without sacrificing data utility for ADR signal detection. In what follows, we present some background knowledge related to this work, including ADR signal detection and privacy-preserving models, followed by a summarization of our previous work [5] on the deficiency of contemporary PPDP models for publishing SRS datasets.

Spontaneously reporting systems and ADR signal detection According to WHO, the definition of ADRs or ADEs is uncomfortable, noxious, unexpected, or potentially harmful reactions resulting from the use of given medications for patients. Usually, an ADR signal (rule) can be represented as an association between symptoms and drugs with some extra conditions, for example, a rule "Avandia, age $>18$ years old $\Rightarrow$ death."

Statisticians have developed various criteria based on the concept of measuring disproportionality or information component (IC) to evaluate the significance of an ADR signal [6]. The most widely adopted disproportionalitybased measurements are Proportional Reporting Ratio (PRR) [7] and Reporting Odds Ratio (ROR) [8]. The PRR measure is used by the U.K. Yellow Card database and UK Medicines and Healthcare products Regulatory Agency (MHRA), while ROR is used by the Netherlands Pharmacovigilance Foundation. All of these measurements can be calculated using a contingency table as shown in Table 1. Table 2 shows some ADR measures and thresholds that commonly used in the pharmacovigilance community for detecting ADR signals.

\section{Privacy models for microdata publishing}

Microdata refer to a kind of data which contains individual information and usually can be represented as tables including tuples defined in a set of attributes, and we can divide these attributes into the following categories:

- Explicit Identifiers (ID): These refer to attributes that can uniquely identify each individual, such as SSN, Name, etc.

- Quasi-identifiers (QID): These refer to attributes that might be linked with external information to re-identify some of the individuals, e.g., Sex, Age, ZIP code, etc.

- Sensitive Attributes (SA): These refer to attributes that contain sensitive information, such as Disease, Salary, etc.

Table 1 The $2 \times 2$ contingency table used for the identification of ADRs

\begin{tabular}{llll}
\hline & Suspected ADR & $\begin{array}{l}\text { Without the } \\
\text { suspected ADR }\end{array}$ & Total \\
\hline Suspected drug & $a$ & $b$ & $a+b$ \\
Other drugs & $c$ & $d$ & $c+d$ \\
Total & $a+c$ & $b+d$ & $\mathrm{~N}=a+b+c+d$ \\
\hline
\end{tabular}


Table 2 Commonly used ADR measures and thresholds

\begin{tabular}{lll}
\hline Measure & Formula & Threshold \\
\hline PRR & $\frac{a /(a+b)}{c /(c+d)}$ & PRR $-1.96 \times \mathrm{SD}>1$ \\
& & PRR $\geq 2, a \geq 3, X^{2} \geq 4$ \\
ROR & $\frac{a / c}{b / d}$ & ROR $-1.96 \times \mathrm{SD}>1$ \\
IC & $\log _{2} \frac{a(a+b+c+d)}{(a+b)(a+c)}$ & Expect $(/ C)-1.96 \times \mathrm{SD}>0$ \\
\hline
\end{tabular}

- Non-sensitive Attributes (NSA): These refer to attributes not fall into the above three categories.

Since Sweeney [9] pointed out that publishing microdata by only removing $I D$ without paying attention to QID may threat the privacy of data owners, there have been a lot of researches on this topic [4]. These research efforts towards protecting released microdata aim at thwarting two primary types of privacy attacks, individual disclosure and attribute disclosure.

Individual disclosure (also known as table linkage attack) refers to the situation that a specific tuple for an individual in the published table is re-identified. The most famous privacy model for this purpose is $k$-anonymity [9]. With $k$-anonymity, the data publisher should generalize QID of the data such that each QID group contains no less than $k$ tuples, making a given record indistinguishable from at least $k-1$ other records by QID. Attribute disclosure (also known as attribute linkage attack) refers to the situation that the sensitive attribute value of an individual can be inferred without the necessity to link the value to a specific tuple. The prevailing model for this protection is $l$-diversity [10], which requires each $Q I D$ group contains at least $l$ "well-represented" sensitive values so as to ensure the probability of inferring the specific sensitive value within each QID group will be no more than $1 / l$.

\section{Problems of contemporary privacy models}

We summarize our previous work on the deficiency of contemporary PPDP models for publishing SRS datasets [5]. First, we present the features of SRS data, and then summarize the results of our analysis.

\section{Special features of SRS data}

- Rare Events: Usually, undiscovered or new ADRs are rarely observed, so almost all criteria used in measuring the significance of ADRs ignore or overlook the frequency of ADRs. For example, the MHRA measure may output a suspected signal even it occurs only three times. With PPDP models, we often generalize or suppress the records, which may increase the risk of false positive as well as false negative signals of ADRs, especially when we perform stratified ADR detection by factors such as Age, Gender, and Location, i.e., members of the typical QID.
- Multiple Individual Records: A typical SRS data usually contains reports called follow-ups, which complement the information of an initial report and have to be merged with the initial report to form a more accurate and complete version. Most of contemporary PPDP models assume that there is only one record for each individual, e.g., $k$-anonymity, $l$-diversity. Overlooking the existence of multiple individual records might impair the privacy requirement to be achieved. For example, consider a table satisfying $k$-anonymity. A QID group might contain $k$ tuples, all of which are of the same individual, thus ruin the privacy requirement.

- Multi-valued Sensitive and Quasi-sensitive Attribute: Quasi-sensitive attributes (QSA) are not sensitive attributes, but as link to external knowledge may reveal sensitive information of an individual. Typical SRS datasets, e.g., FAERS, usually contain Drug and PT (Preferred Terms of symptoms), each of which, if being linked with external knowledge of clinical treatments, could reveal the disease information of an individual. For example, Prezista and Ritonavir are commonly used together for treating HIV; knowing a patient taking these medicines is almost equivalent to perceiving him having HIV. Besides, FAERS contains another attribute named INDI_PT, which records the indications of the patient before treatment. Values of this attribute can be sensitive (represent some disease, e.g., Multiple Sclerosis) or quasi-sensitive (describe symptoms of some illness, e.g., Muscle Spasticity, possibly caused by Parkinson's disease). All of these three attributes are multi-valued, i.e., containing more than one value. Very few PPDP models can handle multi-valued sensitive attributes and consider the existence of quasi-sensitive attributes.

\section{Analysis of previous work}

Our previous work in [5] can be summarized as follows:

(1) Variants of $k$-anonymity or $l$-diversity overlook the existence of rare instances in the dataset.

(2) Only very few models, e.g., $(X, Y)$-privacy [11], consider multiple individual records.

(3) Except QS $l$-diversity [12], no model notices the existence of quasi-sensitive attributes, not to mention the case of multivalued quasi-sensitive attributes.

(4) Most models entail the assumption of single sensitive attribute, while very few embrace the situation of multiple sensitive attributes, e.g., $(\alpha, k)$ anonymity [13], Multi-sensitive $l$-diversity [14].

(5) No model takes into account all of the mentioned features of SRS datasets, which raises the need to design a new PPDP model to handle these features. 


\section{Methods}

\section{The proposed $\mathrm{MS}\left(k, \theta^{*}\right)$-bounding model}

To solve the aforementioned problems, we developed a privacy model called Multi-Sensitive $\left(k, \theta^{*}\right)$-anonymity (abbrev. $\operatorname{MS}\left(k, \theta^{*}\right)$-anonymity). Let $D$ be SRS data to be published that consists of four disjoint sets of attributes, $Q I D, S A, Q S A$, and $N S A$, i.e., $D=<Q I D, S A, Q S A, N S A>$, and $D^{*}$ the released SRS data after anonymization. We called the records with the same QID values a "QIDgroup." We also assume an external knowledge table $E$ about treatment is available, which can be constructed from websites such as Drugs.com, wrongdiagnosis, etc. For simplicity, let $E$ contain a pair of attribute group $\left(Q_{E}, S_{E}\right)$, where $Q_{E}$ denotes the set of attributes that can be linked with QSA in D, e.g., Drug, and $S_{E}$ the set of sensitive attributes, e.g., Disease.

Definition 1 (Confidence). Let $s$ be a sensitive value in $S A$ or $S_{E}$. For a QID-group in $D$ (or $D^{*}$ ) with value of $q$, we define the probability that $q$ have $s$ as

$$
\operatorname{conf}(q \rightarrow s),
$$

and the same probability after linking $E$ via $S A$ and $S_{E}$ as

$$
\operatorname{conf}(q \rightarrow s, E) \text {. }
$$

Definition 2 (Confidence Bounding). Let $S=\left\{s_{1}, s_{2}\right.$, ..., $\left.s_{l}\right\}$ be the set of sensitive values to be protected and $\theta^{*}=\left(\theta_{1}, \theta_{2}, \ldots, \theta_{l}\right)$ be the user specified disclosure probability thresholds associated with $S$, where $\theta_{i}$ denotes the threshold for $s_{i}, 1 \leq i \leq l$. That is, $\theta_{i}$ is an upper bound of the confidence to infer any QID-group having $s_{i}$, with or without external knowledge $E$, i.e.,

$$
\operatorname{conf}\left(q \rightarrow s_{i}, E\right) \leq \theta_{i}
$$

Note that $S$ is a subset of all values legal in $S A$ and $S_{E}$, i.e.,

$$
S \subseteq \mathrm{U}_{A \in S A \cup S_{E}} \operatorname{dom}(A),
$$

where $\operatorname{dom}(A)$ represents the domain of attribute $A$.

Definition $3\left(\mathbf{M S}\left(\boldsymbol{k}, \boldsymbol{\theta}^{*}\right)\right.$-bounding). Given $S$ and the corresponding $\theta^{*}$, we say a release data $D^{*}$ satisfies $\operatorname{MS}(k$, $\left.\theta^{*}\right)$-bounding if

(1)Every QID-group contains at least $k$ distinct individuals (cases);

(2)The confidence to infer any QID-group $q$ having $s_{i}$ is less than $\theta_{i}$, i.e., $\operatorname{conf}\left(q \rightarrow s_{i}, E\right) \leq \theta_{i}$.

In $\operatorname{MS}\left(k, \theta^{*}\right)$-bounding, we define $\theta^{*}$ to control the ratio of sensitive values in QID group because not all sensitive values is "really" sensitive. For example, most diseases are sensitive for people, but it does not matter when the others know someone got a flu. This model can solve the multiple individual records problem because $k$ is defined by the distinct individuals, and it is easy to check whether the QID group satisfies the model or not.

Another noteworthy thing is about the setting of confidence bounding $\theta^{*}$. In general, as applying $\operatorname{MS}\left(k, \theta^{*}\right)$ bounding to the dataset, every $\theta_{i}$ in $\theta^{*}$ should be no less than the frequency of $s_{i}$ in the dataset, i.e., we must set every $\theta_{i}$ so as to satisfy $\theta_{i} \geq P\left(s_{i}\right)$. This is because after generalization the occurrence of $s_{i}$ in every QID-group is no less than $P\left(s_{i}\right)$, and so setting $\theta_{i}<P\left(s_{i}\right)$ nullifies the work of anonymization, i.e., the result fails to meet the privacy requirement. However, for a dataset containing some relatively frequent sensitive values, we still can apply $\operatorname{MS}\left(k, \theta^{*}\right)$-bounding to the dataset using some other methods like adding counterfeit records or suppressing some of those sensitive values, though those method may severely decrease the utility of the data.

Example 1. Table 3 illustrates a sample of the FAERS data, where ISR and CaseID denote the IDs of a record and an event, respectively. Since an event may have more than one reporting records, a CaseID can correspond to many different ISRs. Here we assume QID comprises \{Age, Gender, Country\}. Table 3(a) shows the anonymized table $D^{*}$ composed of two QID groups, ([20-30], M, USA) and ([30-40], F, UK), each of which contains two different events; Table 3(b) represents the external table $E$ showing knowledge of treating diseases with drugs. It is not hard to derive that the probability of each disease associated with a specific QID group is less than 0.4, e.g., conf $([30-40], \mathrm{F}$, UK $\rightarrow$ Headache, $E)=0.25$. This anonymized table $D^{*}$ thus satisfies $\mathrm{MS}(2,0.4)$-bounding.

\section{Anonymization algorithm for $\mathrm{MS}\left(k, \theta^{*}\right)$-anonymity Algorithm basics}

Our algorithm is a hybrid of greedy and clustering approaches. We view each QID-group as a cluster and

Table 3 A sample FAERS data satisfying MS(2, 0.4)-bounding

(a) Anonymized table

$\begin{array}{llllll}\text { ISR } & \text { CaselD } & \text { Age } & \text { Gender } & \text { Country } & \text { Drugs } \\ 001 & 001 & {[20-30]} & M & \text { USA } & \text { Paracetamol } \\ 002 & 001 & {[20-30]} & M & \text { USA } & \text { Paracetamol } \\ 003 & 002 & {[20-30]} & M & \text { USA } & \text { Intron A, Antacid } \\ 004 & 002 & {[20-30]} & M & \text { USA } & \text { Intron A, Antacid } \\ 005 & 003 & {[30-40]} & F & \text { UK } & \text { Paracetamol } \\ 006 & 004 & {[30-40]} & F & \text { UK } & \text { Antacid }\end{array}$

(b) External table

$\begin{array}{ll}\text { Drug } & \text { Diseases } \\ \text { Asprin } & \text { Flu, Headache, Fever } \\ \text { Intron A } & \text { Hepatitis B, Hepatitis C, Leukemia, Melanoma } \\ \text { Paracetamol } & \text { Headache, Fever } \\ \text { Antacid } & \text { Stomachache, GERD }\end{array}$


so develop a clustering-based method [15] to form all QID-groups.

Each QID-group (cluster) begins with a randomly selected record, and then is gradually increased by adding an isolated record that exhibits the best characteristic among all candidates. This process continues until the group is composed of $k$ different cases. Finally, the QIDs of all records belonging to the same cluster are generalized to the same value.

We use generalization rather than suppression as the anonymization operation because suppression tends to remove records corresponding to rare events. We adopt both hierarchy-based generalization and hierarchy-free generalization; the former is used when a value generalization hierarchy is defined for the attribute (usually, it is categorical), otherwise the latter is used. For example, we adopt the age hierarchy defined in $\mathrm{MeSH}$ [16], a domain knowledge of value generalization hierarchies widely used in medical and healthcare areas. In the following, we describe the metric for evaluating an isolated record quality.

Intuitively, the best record to be included into a QIDgroup should exhibit the most similarity to the group. This implies its addition will result in the least degree of generalization (distortion of data, destruction of utility, or information loss) to be performed on the QID attributes of the group. Here in this study, we adapt the measure of information loss defined in [15].

Definition 4 (Information Loss). Let $g$ denote a group (cluster) constructed during the execution of our algorithm, where the QID comprise two different sets, numerical attributes $N_{1}, N_{2}, \ldots, N_{m}$, and categorical attributes $C_{1}, C_{2}, \ldots, C_{n}$, and each $C_{i}$ is associated with a generalization hierarchy $T_{i}$. The information loss $(I L)$ of group $g$ is defined as follows:

$$
I L(g)=|g| \times\left(\sum_{i=1}^{m} \frac{\max \left(N_{i}, g\right)-\min \left(N_{i}, g\right)}{\max \left(N_{i}\right)-\min \left(N_{i}\right)}+\sum_{j=1}^{n} \frac{h\left(C_{j}, g\right)}{h\left(C_{j}\right)}\right),
$$

where $\max \left(N_{i}\right)\left(\min \left(N_{i}\right)\right)$ and $\max \left(N_{i}, g\right)\left(\min \left(N_{i}, \mathrm{~g}\right)\right)$ denote the maximum (minimum) values of attribute $N_{i}$ in the whole dataset and group $g$, respectively; $|g|$ denotes the number of records in $g ; h\left(C_{j}\right)$ is the height of the hierarchy tree $T_{j}$, and $h\left(C_{j}, g\right)$ the height of the generalized value of $C_{j}$ for all records in $g$, i.e., the lowest common ancestor in $T_{j}$ with respect to every $C_{j}$ value in $g$.

The information loss measures how generalization impact the data utility. As we are building a group $g$ by adding new records, we can use the difference of $I L$ $(\Delta I L)$ between the original group and the group with record $r$ to determine the best record that produces the least $\Delta I L$, i.e.,

$$
\Delta I L(g, r)=I L(g \cup\{r\})-I L(g),
$$

and the best choice $r_{\text {bst }}$ is

$$
r_{\mathrm{bst}}=\operatorname{argmin}_{r} \Delta I L(g, r) .
$$

In addition to the data distortion, the privacy requirement is another factor critical for the determination of the best record. This is because the inclusion of a new record would increase the disclosure risk of some sensitive values in the resulting $Q I D$-group. We introduce a new parameter called Privacy Risk $(P R)$ to measure the risk of sensitive value disclosure incurred by adding new records into the QID-group, thus alleviating the breach of our privacy requirement.

Let $S_{r}$ denote the set of sensitive values contained in record $r$. Consider a QID-group $g$ and a sensitive value $s \in S_{r}$. Let $\sigma_{s}(g)$ represent the number of records in $g$ containing sensitive value $s$. We define the maximum number of records in $g, \eta_{s}(g)$, that will cause the breach of the bound $\theta_{s}$ associated with $s$

$$
\eta_{s}(g)=\left\lfloor\max \{k,|g|\} \times \theta_{s}\right\rfloor,
$$

and the privacy risk to explore $s$ with the inclusion of record $r$ as

$$
P R_{s}(g \cup\{r\})= \begin{cases}\frac{\sigma_{s}(g)}{\eta_{s}(g \cup\{r\})-\sigma_{s}(g)} & \text { if } \eta_{s}(g \cup\{r\})>\sigma_{s}(g) \\ \infty & \text { otherwise }\end{cases}
$$

Since a record may contain multiple sensitive values, the privacy risk of group $g$ caused by including $r$ can be defined as the summation of the risk to each sensitive value.

\begin{tabular}{|c|c|c|c|c|}
\hline CaselD & Gender & Age & Weight & Indications \\
\hline r1 & Male & Young Adult & {$[50-75]$} & 11 \\
\hline r2 & Male & Young Adult & [50-75] & $12,13,14$ \\
\hline r3 & Male & Young Adult & {$[50-75]$} & 12,13 \\
\hline$r 4$ & Male & Young Adult & {$[50-75]$} & 12 \\
\hline \multicolumn{5}{|c|}{ (b) Isolated records } \\
\hline CaselD & Gender & Age & Weight & Indications \\
\hline$r 5$ & Male & Adocent & 50 & 11 \\
\hline r6 & Female & Adult & 40 & 13,14 \\
\hline r7 & Male & Young Adult & 80 & 12,13 \\
\hline
\end{tabular}

Definition 5 (Privacy Risk). Let $g$ denote a group (cluster) constructed during the execution of our

Table 4 An example data

(a) A known group $g$ 


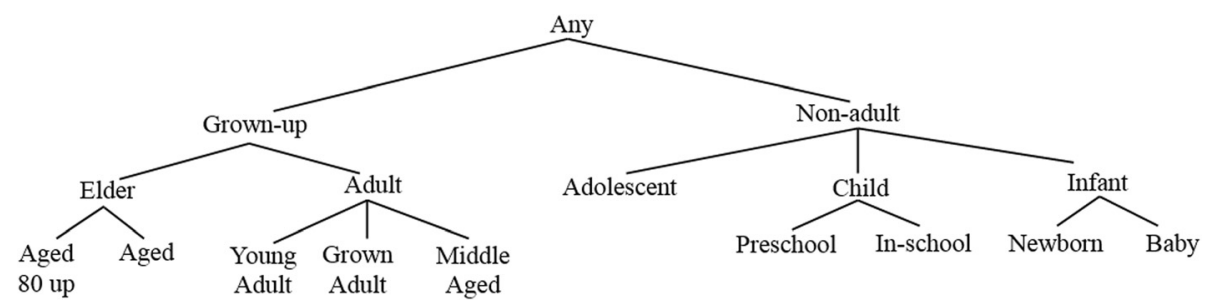

Fig. 1 Value hierarchy for age

algorithm. The privacy risk $(P R)$ to group $g$ caused by including a record $r$ is

$$
P R(g, r)= \begin{cases}1+\sum_{s \in S_{r}} P R_{s}(g \cup\{r\}) & \text { if } \eta_{s}(g \cup\{r\})>\sigma_{s}(g) \\ \infty & \text { otherwise }\end{cases}
$$

Finally, we refine $\Delta I L$ into $\Delta I L$ ' as follows:

$$
\Delta I L^{\prime}(g, r)=\Delta I L(g, r) \times P R(g, r),
$$

and

$$
r_{\mathrm{bst}}=\operatorname{argmin}_{r} \Delta I L^{\prime}(g, r) .
$$

Note that when all sensitive values in $S_{r}$ are new to group $g, \sigma_{s}(g)=0$ and so is $P R(g, r)$, which will dismiss the effect contributed by information loss $(\Delta I L)$. To avoid this situation, we add an increment into (6).

Example 2. Consider Table 4 which consists of a group of four cases, r1 to r4, with $g=\{$ Male, Young Adult, 50$75\}$ and three isolated cases, r5 to r7. Figure 1 shows the age hierarchy defined in $\mathrm{MeSH}$ and Fig. 2 depicts a simple hierarchy for gender. Let $Q I D=\{$ Gender, Age, Weight $\}$ and $S A=\{$ Indications $\}$, and suppose $k=5, \theta^{\prime \prime}=0.6$, and weight range $=0 \sim 100$. The information loss for group $g$ is

$$
I L(g)=4 \times\left(\frac{0}{1}+\frac{0}{2}+\frac{75-50}{100-0}\right)=4 \times 0.25=1,
$$

and for $g \cup\{\mathrm{r} 5\}, g \cup\{\mathrm{r} 6\}$, and $g \cup\{\mathrm{r} 7\}$ the values are

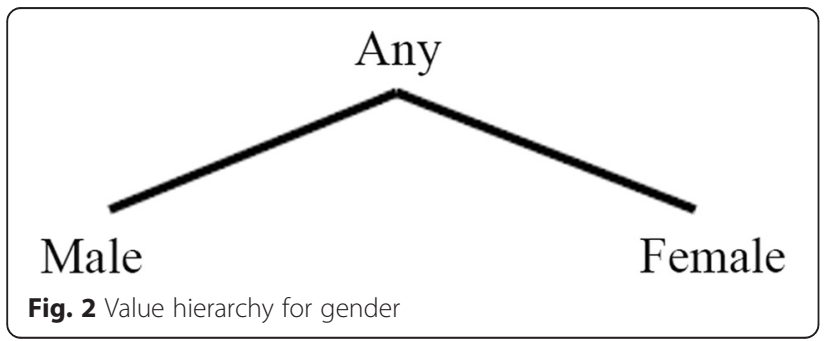

$$
\begin{aligned}
I L(g \cup\{r 5\}) & =5 \times\left(\frac{0}{1}+\frac{2}{2}+\frac{75-50}{100-0}\right) \\
& =5 \times(1+0.25)=6.25 \\
I L(g \cup\{r 6\}) & =5 \times\left(\frac{1}{1}+\frac{1}{2}+\frac{75-40}{100-0}\right)=9.25 \\
I L(g \cup\{r 7\})= & \times\left(\frac{0}{1}+\frac{0}{2}+\frac{85-50}{100-0}\right)=5 \times(0.35)=1.75
\end{aligned}
$$

Next, it is easy to compute the $\Delta I L s$.

$$
\begin{aligned}
& \Delta I L(g, \mathrm{r} 5)=6.25-1.4=4.85 \\
& \Delta I L(g, \mathrm{r} 6)=7.25-1.4=5.85 \\
& \Delta I L(g, \mathrm{r} 7)=1.75-1.4=0.35
\end{aligned}
$$

The privacy risks are

$$
\begin{aligned}
& P R(g, \mathrm{r} 5)=1+\frac{1}{3-1}=1.5 \\
& P R(g, \mathrm{r} 6)=1+\frac{2}{3-2}+\frac{1}{3-1}=3.5 \\
& P R(g, \mathrm{r} 7)=\infty
\end{aligned}
$$

Finally, we can compute the $\Delta I L$ 's and obtain the best choice $r_{\mathrm{bst}}$ among $\mathrm{r} 5$ to $\mathrm{r} 7$, concluding $r_{\mathrm{bst}}=\mathrm{r} 5$.

$$
\begin{aligned}
r_{\text {bst }} & =\operatorname{argmin}_{r}\left\{\Delta I L^{\prime}(g, \mathrm{r} 5), \Delta I L^{\prime}(g, \mathrm{r} 6), \Delta I L^{\prime}(g, \mathrm{r} 7)\right\} \\
& =\operatorname{argmin}_{r}\{4.18 \times 1.5,5.85 \times 3.5, \infty\}=\mathrm{r} 5
\end{aligned}
$$

\section{Detail description}

Algorithms 1 and 2 present the description of our algorithm, which is composed of two stages. The first stage is to create as many $Q I D$-groups that satisfy $\operatorname{MS}\left(k, \theta^{\prime \prime}\right)$ bounding as possible. We introduce a concept called combined record (or super record) to handle the issue of multiple individual records. That is, all records with the same CaseID are combined into a super record before the anonymization procedure. This avoids the abnormal situation that members of this CaseID group will be, after generalization, divided into different $Q I D$-groups, which will cause larger bias on the data quality and perplex the process of identifying duplicate records during ADR signal detection. 


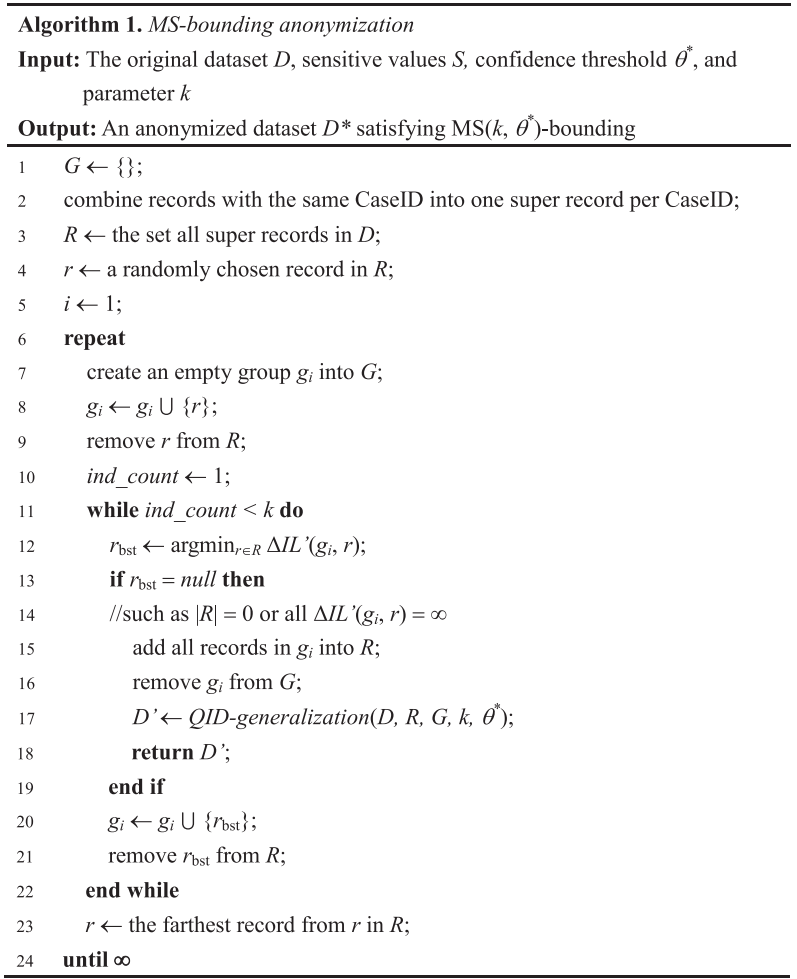

Initially, we combine records with the same CaseID into one super record per CaseID by generalizing the values of those records. The generalization is necessary because not all members of the same CaseID exhibit the same $Q I D$ value. This is due to the existence of follow-up records, which represent compensation for the initial report and so may contain update information.

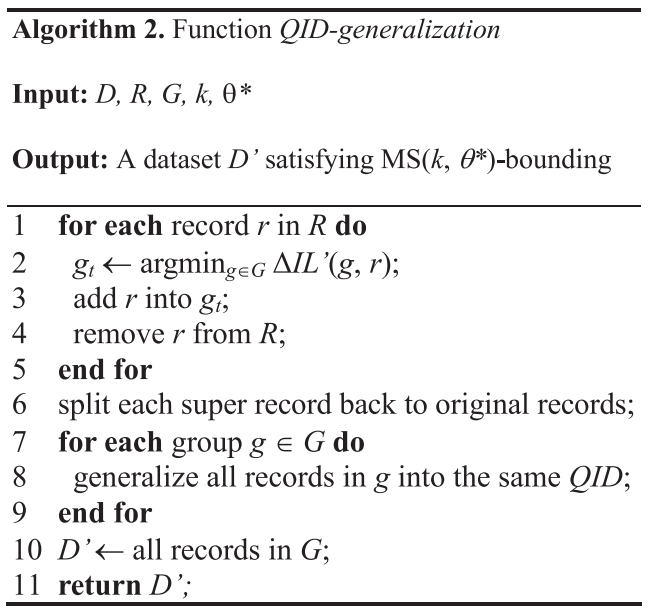

Next, we create an empty group and add into it a randomly selected record, then into which we add more records step by step, each with the least $\Delta I L$ ' (defined in (7)) until the group satisfies $\mathrm{MS}\left(k, \theta^{*}\right)$-bounding. Thirdly, we choose a new record that is most distinguished from the one chosen for creating the latest group and repeat the same steps to grow the group. These steps are repeated until the remaining records cannot form a group, e.g., the number of records is less than $k$ or most of the remaining records contain the same sensitive value.

The second stage is then activated by calling function QID-generalization (see Algorithm 2). First, we take care of the ungrouping records by adding each of them into the group that produces the least $\Delta I L^{\prime}$ to ensure the utility and meet the privacy requirement. Next, we split those combined records back to their original records (do not change the group they belong to). Finally, we generalize all records within the same group into the same QIDs such that the whole data set will satisfy $\operatorname{MS}\left(k, \theta^{*}\right)$-bounding.

\section{Results and Discussions}

We have conducted a series of experiments to confirm if our model is more suitable for anonymizing SRS datasets than prevailing PPDP models. We describe the design of each experiment, present the experimental results, and state our observations.

\section{Experimental design}

All experiments were conducted over FAERS datasets, which is a SRS system provided by U.S. Food and Drug Administration (FDA) and released quarterly. Each report in FAERS is uniquely identified by an attribute called ISR, and contains an attribute CaseID to identify distinct individuals, along with some demographic information such as Weight, Age, and Gender, drugs information such as drug name (Drug) and indication (INDI_PT), and reaction information $(P T)$.

We used \{Weight, Age, Gender\} as QID, CaseID as the individual identifier, and used drug indication (INDI_PT) and drug reaction $(P T)$ as $S A$. Datasets from 2004Q1 to 2011Q4 were selected to build the test sets, where any record with $Q I D$ containing missing values was discarded.

Four prevailing PPDP models were evaluated against our model. They are $k$-anonymity, $(X, Y)$-anonymity, Multi-sensitive $l$-diversity, and $(\alpha, k)$-anonymity. These models were chosen because each of them is the representative or the prevailing models, and can be applied to anonymize SRS data without additional modification; this is why $l$-diversity is replaced by Multi-sensitive $l$-diversity.

All models were evaluated from two aspects: the quality of resulting anonymized dataset, measured by two 
criteria, i.e., data utility and privacy risk, and the influence to ADR signals.

For the anonymization quality, we considered two measurements. The first one called Normalized Information Loss (NIL) is used to measure the data utility, defined as follows:

$$
\operatorname{NIL}\left(D^{*}\right)=\frac{1}{n_{g} \times|Q I D|}\left(\sum_{g \in D^{*}} I L(g)\right),
$$

where $D^{*}$ is the anonymized data table, $n_{g}$ denotes the number of QID-groups in $D^{* \prime}, g$ denotes a $Q I D$-group, and $|Q I D|$ the cardinality of $Q I D$. The value of NIL ranges over $[0,1]$; larger NIL means poorer data utility.

The second one called Dangerous Ratio $(D R)$ is used to measure the privacy risk of anonymized dataset, defined as follows:

$$
D R=\frac{\text { number of dangerous } Q I D-\text { groups }}{\text { number of } Q I D-\text { groups }}
$$

A QID-group is dangerous if it contains at least one unsafe sensitive value, that is, the attacker's confidence for inferring that value is higher than the specified threshold. In this sense, the $D R$ measure also estimates the privacy-preserving quality of an anonymized table.

For the influence to ADR signals, we inspect the impact of anonymized data on the strength of observed ADR rules. Following our previous work in [17], we chosen from FDA MedWatch [18] all significant ADR rules that render withdrawal or warning of the drugs and associated with patient demographics, such as age or gender conditions. Detail description of these ADR rules is shown in Table 5 .

Since our model allows non-uniform settings of confidence bounding, i.e., $\theta^{*}$, we considered three different scenarios of thresholds for $\theta^{*}$ to inspect the effect of

\begin{tabular}{lllll} 
Table 5 & Selected ADR rules from FDA MedWatch & \\
\hline $\begin{array}{l}\text { Drug } \\
\text { name }\end{array}$ & Adverse reaction & $\begin{array}{l}\text { Demographic } \\
\text { condition }\end{array}$ & $\begin{array}{l}\text { Marked } \\
\text { year }\end{array}$ & $\begin{array}{l}\text { Withdrawn or } \\
\text { warning year }\end{array}$ \\
\hline AVANDIA & $\begin{array}{l}\text { Myocardial infarction } \\
\text { Death }\end{array}$ & $18 \sim$ & 1999 & 2010 \\
& $\begin{array}{l}\text { Cerebrovascular } \\
\text { accident }\end{array}$ & & & \\
TYSABRI & $\begin{array}{l}\text { Progressive multifocal } \\
\text { leukoencephalopathy }\end{array}$ & $18 \sim$ & 2004 & 2005 \\
ZELNORM & $\begin{array}{l}\text { Cerebrovascular } \\
\text { accident }\end{array}$ & Female & 2002 & 2007 \\
WARFARIN & Myocardial infarction & $60 \sim$ & 1940 & 2014 \\
REVATIO & Death & $\sim 18$ & 2008 & 2014 \\
\hline
\end{tabular}

different settings: 1 ) Uniform setting for $\theta^{*}$, i.e., all confidences of symptoms were set to the same value $(0.2$ or $0.4)$; 2) Level-wise specification, that is, all symptoms (or diseases) were classified into three levels, high sensitive, low sensitive, and non-sensitive. Those symptoms corresponding to high sensitive are assigned a smaller threshold, i.e., 0.2, low sensitive are assigned a larger threshold, i.e., 0.4, and non-sensitive are assigned to $1 ; 3$ ) Frequency-based strategy, the threshold of each symptom is determined based on the idea: "The more frequently the symptom occurs, the less sensitive it is."

\section{Results for uniform confidence setting}

We assume every symptom is of the same sensitivity with confidence bounded by 0.2 or 0.4 . Analogously, $\alpha$ is set to 0.2 or 0.4 for $(\alpha, k)$-anonymity, while $k($ or $l)=5,10,15,20$ for $k$-anonymity, $(X, Y)$-anonymity, Multi-sensitive $l$-diversity, and our $\operatorname{MS}\left(k, \theta^{*}\right)$-bounding. First, we compared the data utility generated by each anonymization method. Figures 3 and 4 show the resulting NILs for every method, where MS $l$-diversity means Multi-sensitive $l$-diversity. Panels inside are for better view of data position, and applicable to all following figures. Since the results for $k$ (or $l$ ) $=10,15$ are somewhere in between those for $k($ or $l)=5,20$ and conform to the overall trend, hereafter we omit these two cases. From the obtained results, we observe that

1. $k$-anonymity and $(X, Y)$-anonymity are good at preserving the data utility, and both exhibit nearly identical results, which are less than those generated by our methods.

2. $(\alpha, k)$-anonymity and our model yield similar NIL results, because under uniform setting the only difference between $(\alpha, k)$-anonymity and our model is that $(\alpha, k)$-anonymity does not consider duplicate reports, yielding not too much effect in information loss. Furthermore, $(\alpha, k)$-anonymity and our model suffer from much less information loss when the confidence threshold is set relatively higher (0.4 vs 0.2 ).

3. Multi-sensitive $l$-diversity causes much more information loss than the other models because the top-down method tends to create larger QID-groups than that by bottom-up method.

4. Even in larger threshold setting, the information loss generated by our method is around 5 to 40 times of that by $k$-anonymity and $(X, Y)$-anonymity, though the values are still small, normally between 0.01 to 0.2 ; the larger $k$ value is, so is NIL. That it, the data utility decreases as larger QID-group is allowed.

It is noteworthy that some datasets anonymized by our method with lower $\theta$ produce very high NILs, i.e., 2004Q4, 2007Q1, and 2010Q3. After further inspection 


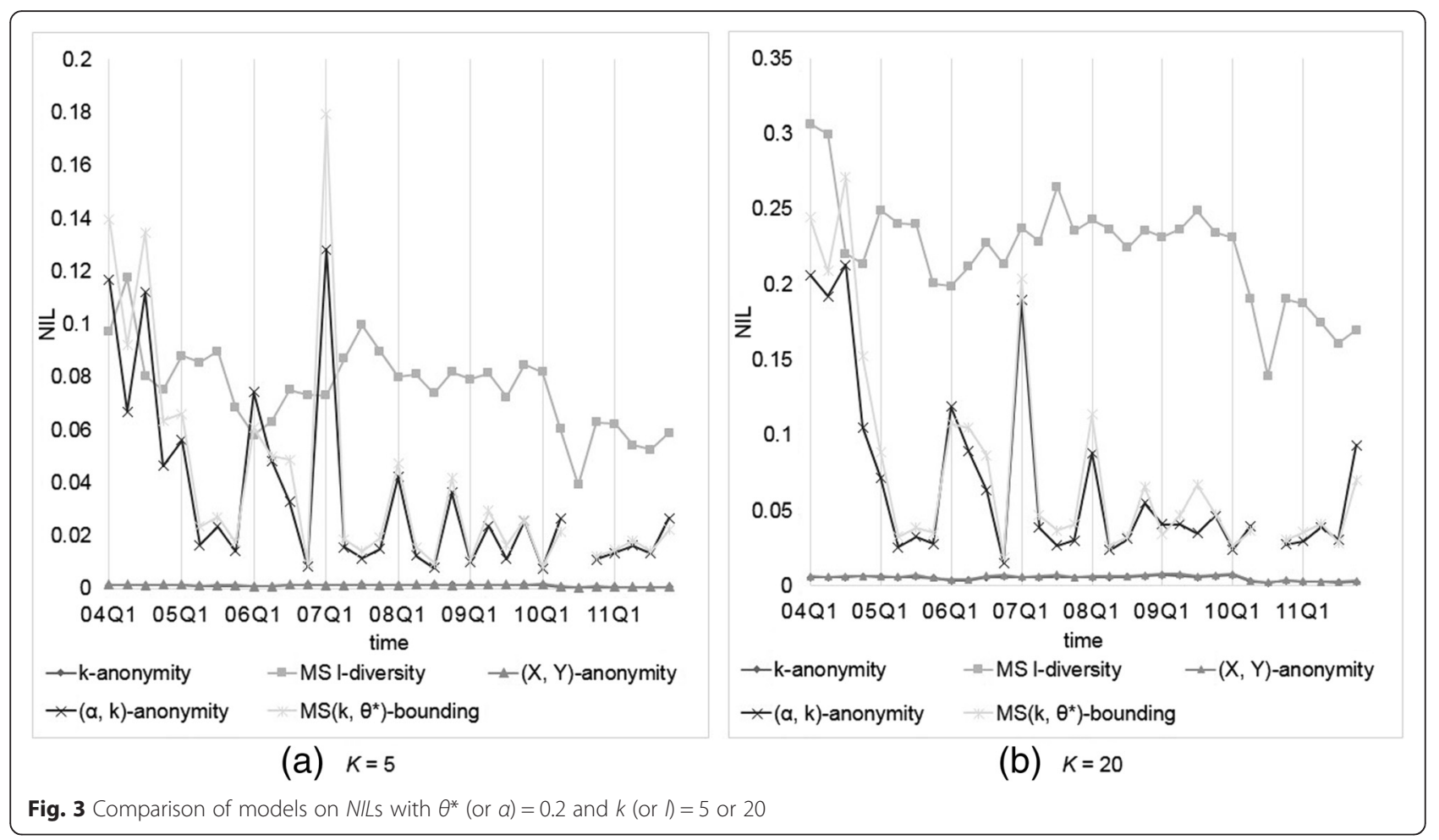

we found that it is because most of these datasets contain some relatively high frequent symptoms. For example, there are 22,730 reports (without missing values) in $2007 \mathrm{Q} 1$, and 3,890 (17.1\%) of them recorded "Diabetes Mellitus Non-Insulin-Dependent," and in 2010Q3,
12,833 of 63,838 (20.1 \%) reports containing "Smoking Cessation Therapy." In this situation, it is hardly to apply $(\alpha, k)$-anonymity or our $\operatorname{MS}(k, \theta)$ )-bounding with $\alpha$ (so as $\theta)=0.2(<20.1 \%)$ to this dataset. It looks like uniform threshold setting of our model is not suitable to data with

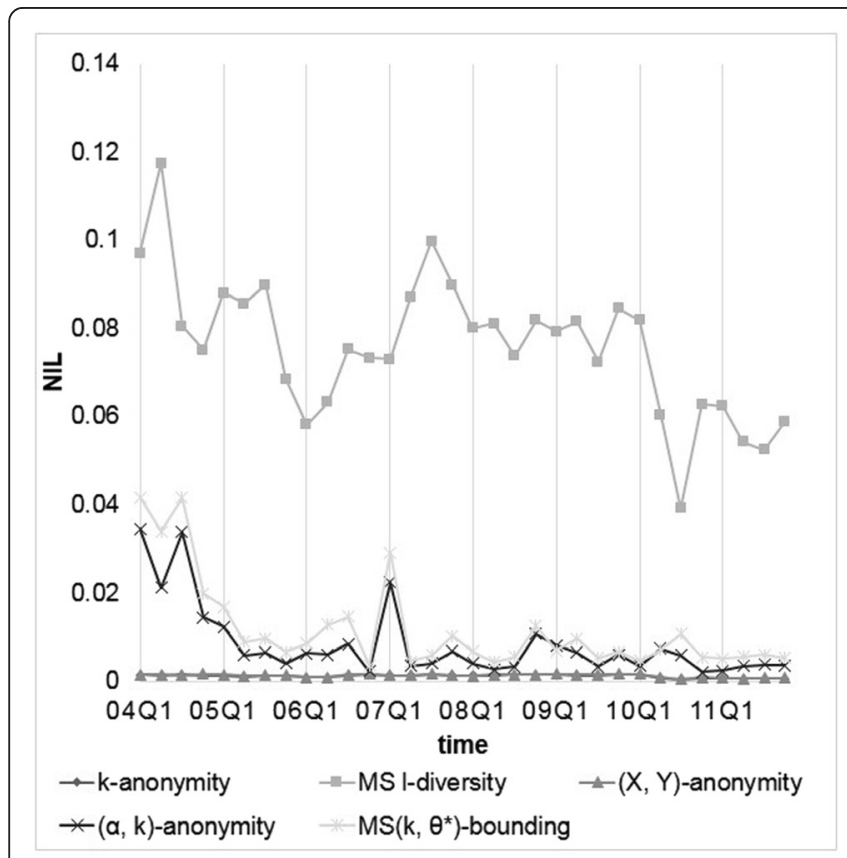

(a) $k=5$

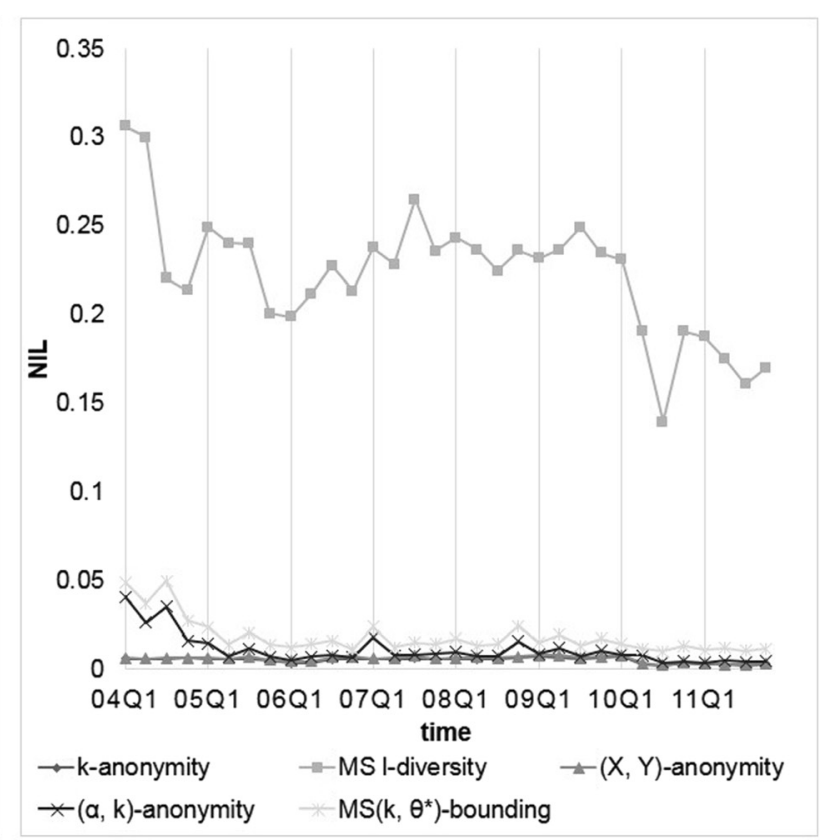

(b) $k=20$

Fig. 4 Comparison of models on NILs with $\theta^{*}$ (or $\left.a\right)=0.4$ and $k$ (or $\left.I\right)=5$ or 20 
high frequent sensitive values, but in most scenarios, the more frequent the values occur, the lesser sensitive they are. All we need is to adopt non-uniform setting for $\theta^{*}$, as to be shown later.

Next, we compared the privacy risk raised by each anonymization method. Figures 5 and 6 depict the resulting $D R$ s for all methods. From the obtained results, we observe that

1. Our $\operatorname{MS}\left(k, \theta^{*}\right)$-bounding yields no $D R$ because the flexibility to set $\theta^{*}$ according to user requirement. On the contrary, $(\alpha, k)$-anonymity would suffer from some DRs because QID-groups contain duplicate reports, which may decrease actual group size, causing violation of the privacy requirement. While $k$ is getting larger, the probability of duplicate reports accumulated to the same group is increasing, further aggravating $D R$ s.

2. Multi-sensitive $l$-diversity does not perform well on protecting the sensitive values. This is because it only guarantees the number of records with distinct sensitive values in each group no less than $l$, which may fail to thwart the attacker's confidence on inferring the patient symptoms.

For those models not considering confidence threshold on sensitive values, including $k$-anonymity and $(X$, $Y$ )-anonymity, it can be observed that the larger $k$ is, the lower $D R$ being generated. That it, the data privacy risk increases as larger QID-group is allowed.

\section{Results for level-wise confidence setting}

To inspect the applicability of our model to more practical situation, we also adopted level-wise setting of $\theta^{*}$. In practice, most symptoms or indications are not "really" sensitive. We choose group of symptoms called "Acquired immunodeficiency syndromes" (a High Level Term (HLT) in MedDRA), which contains 32 PTs and most of them are similar to AIDS, as "high sensitive" with confidence threshold $=0.2$, and another two groups called "Coughing and associated symptoms" and "Allergies to foods, food additives, drugs and other chemicals," which contain 44 PTs, as "non-sensitive" symptoms with confidence threshold $=1$. The confidence thresholds of symptoms not belonging to the above groups are set to 0.4 .

We compared our $\operatorname{MS}\left(k, \theta^{*}\right)$-bounding with those models considering sensitive values, including Multisensitive $l$-diversity and $(\alpha, k)$-anonymity. The parameter setting is $\alpha=0.2$ and 0.4 , and $k$ (or $l)=5,10,15$, and 20 . Figures 7 and 8 show the resulting NILs and DRs, respectively. From the obtained results, we observe that

1. All NILs generated except by $\operatorname{MS}\left(k, \theta^{*}\right)$-bounding are the same as observed previously, but DRs are different because of various threshold settings.

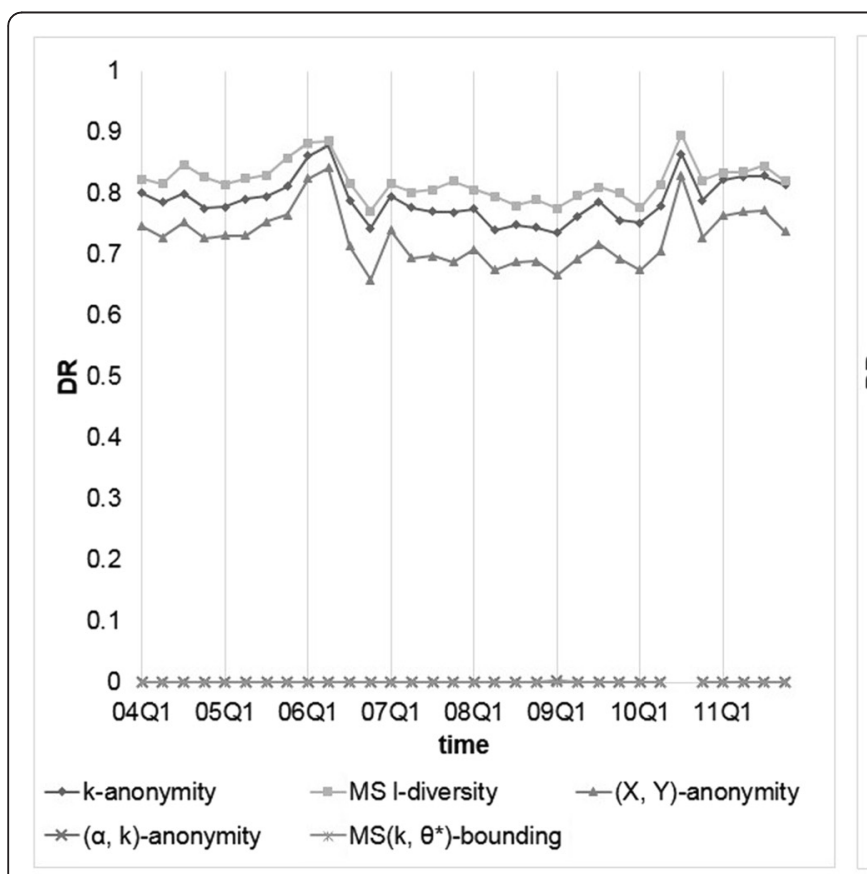

(a) $k=5$

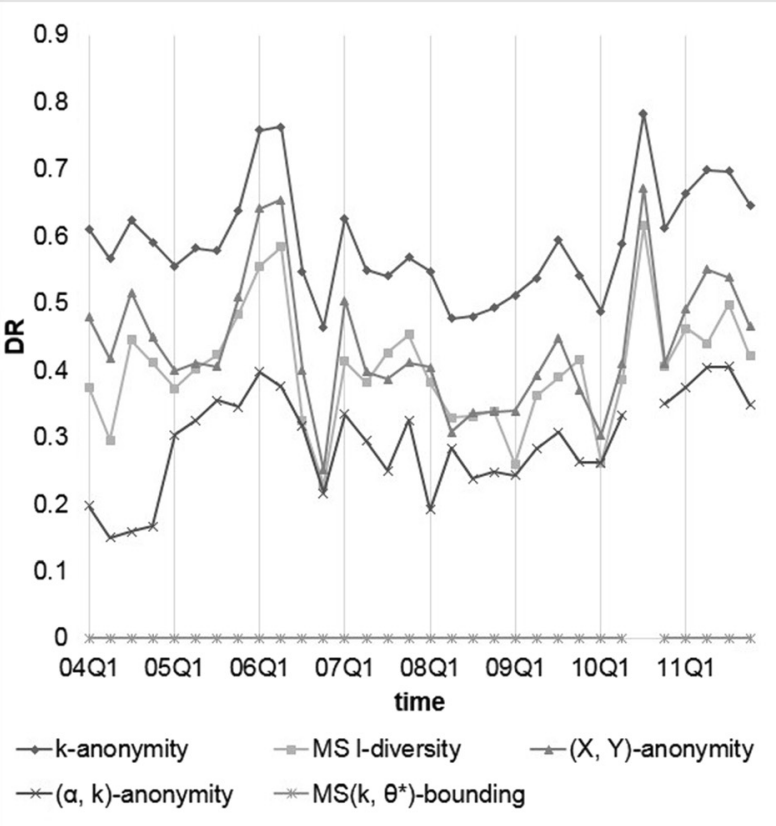

(b) $k=20$

Fig. 5 Comparison of models on DRs with $\theta^{*}$ (or $\left.a\right)=0.2$ and $k$ (or $\left.I\right)=5$ or 20 


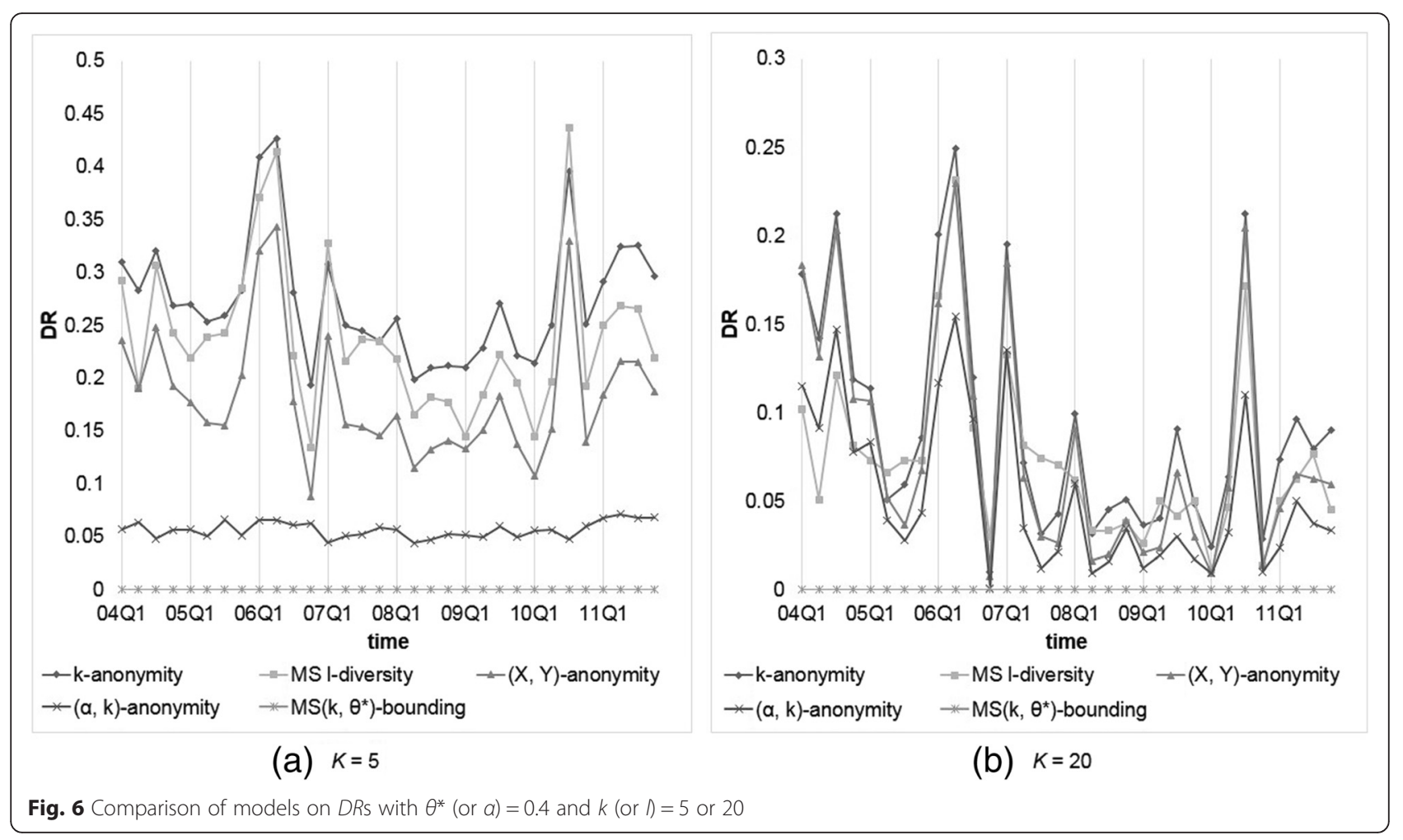

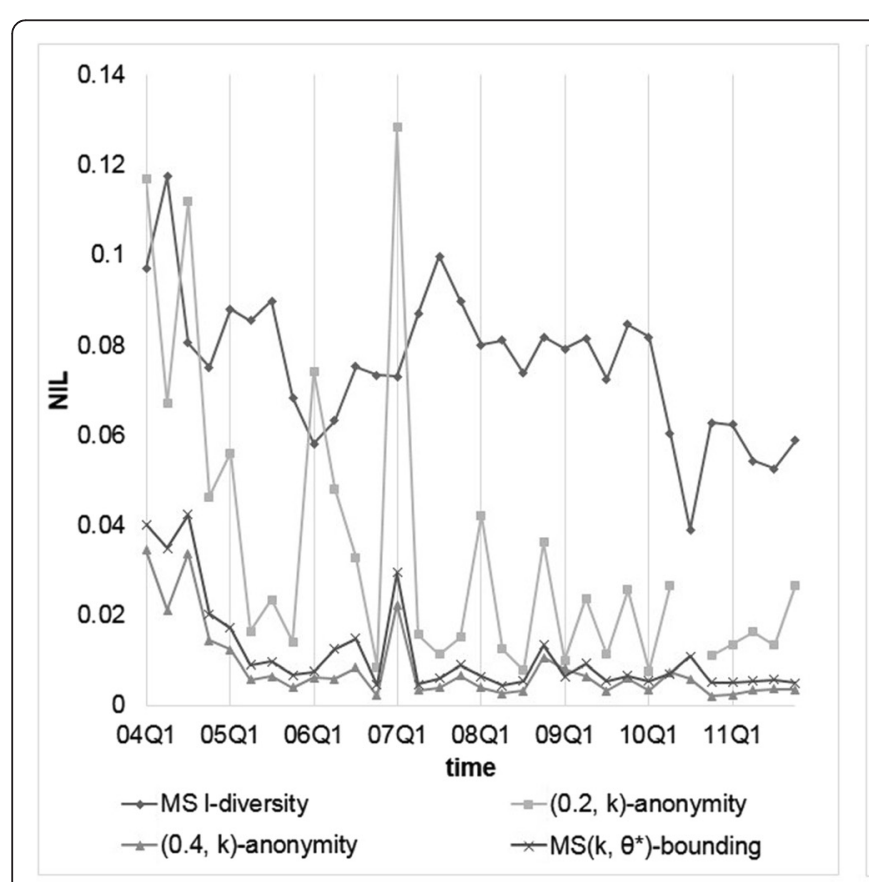

(a) $k=5$

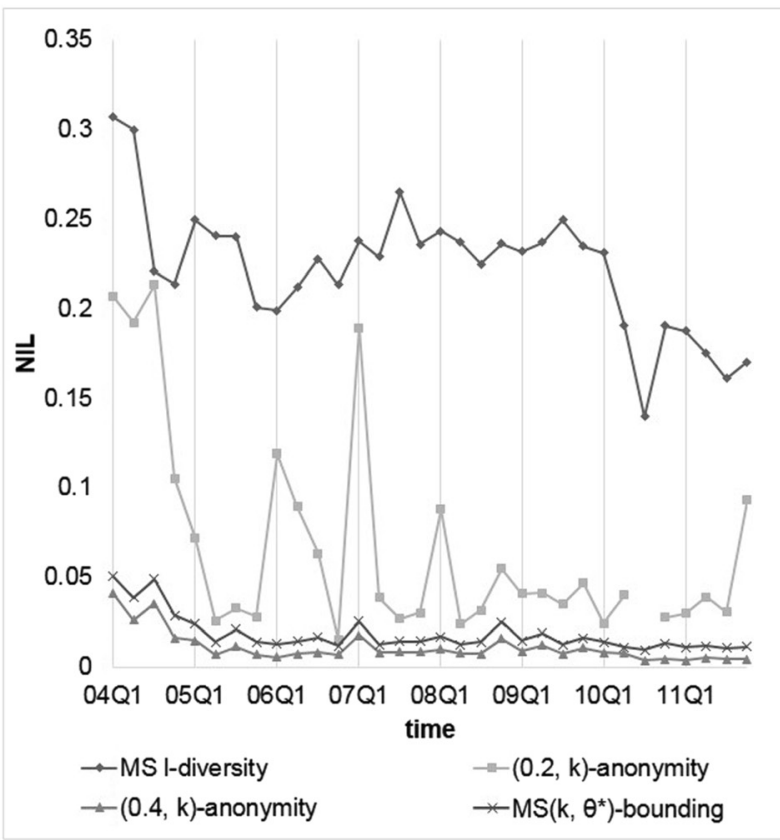

(b) $k=20$

Fig. 7 Comparison of our MS( $\left(\theta^{*}\right)$-bounding with level-wise setting with other models on NILS with $k$ (or $\left.I\right)=5$ or 20 


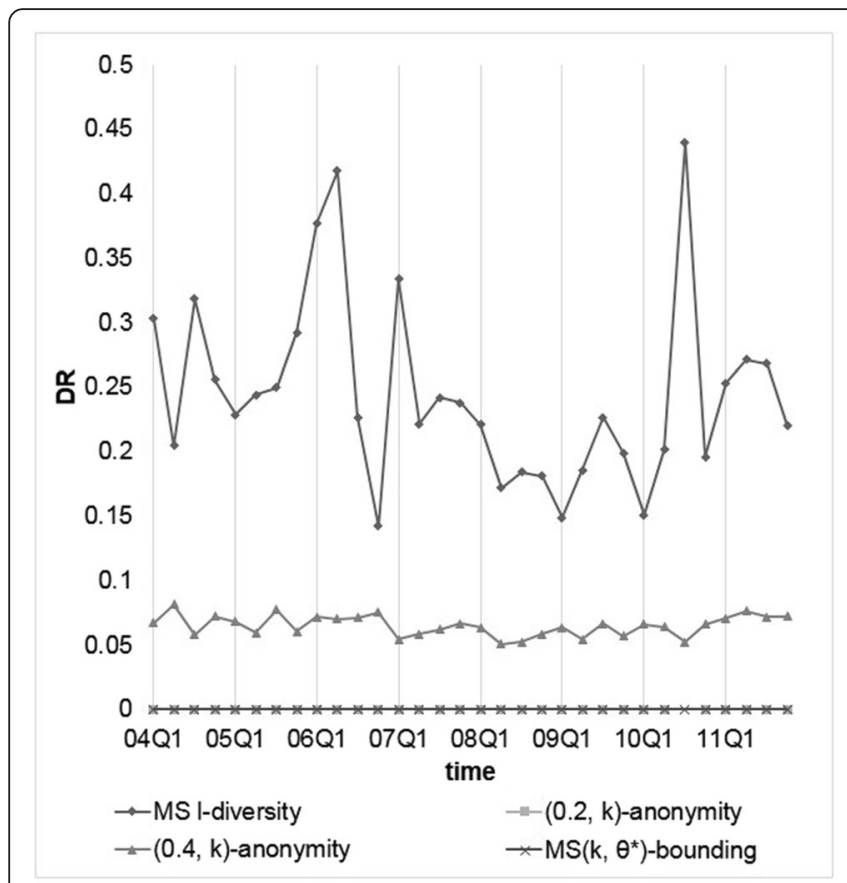

(a) $k=5$

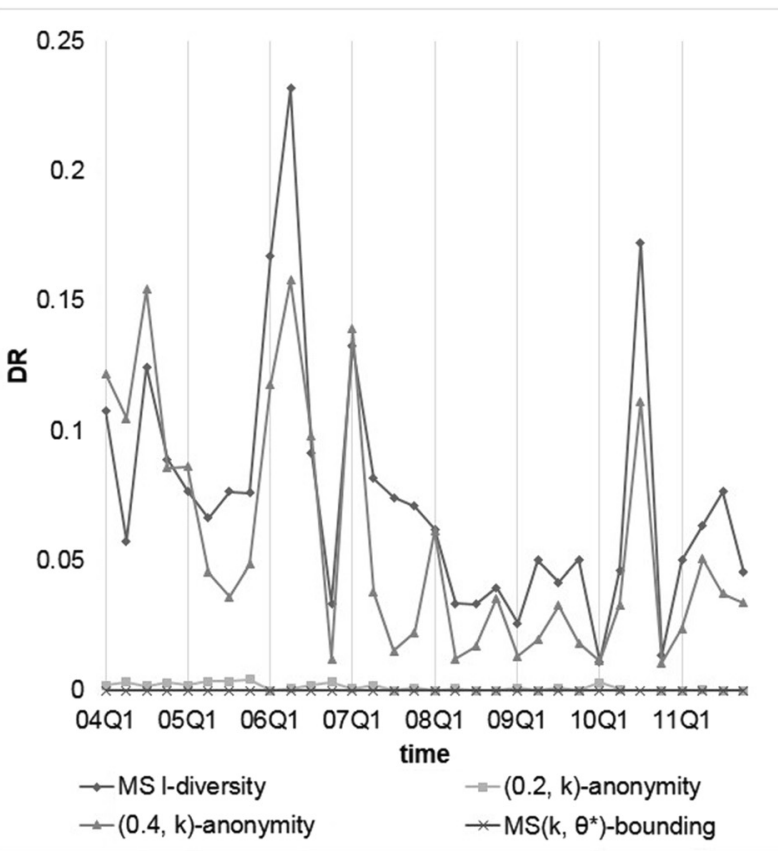

(b) $K=20$

Fig. 8 Comparison of our $\mathrm{MS}\left(k, \theta^{*}\right)$-bounding with level-wise setting with other models on DRs with $k$ (or $\left.l\right)=5$ or 20

2. Because records containing "high sensitive" and duplicate records are rare, $(0.2, k)$-anonymity generate very few DRs. However, the generated NIL is very high for data with high frequent sensitive value that may decrease data utility severely.

3. $\operatorname{MS}\left(k, \theta^{*}\right)$-bounding produces only a little larger NIL than $(0.4, k)$-anonymity because in this level-wise specification, most symptoms receive confidence threshold at 0.4 . In contrast, $\operatorname{MS}\left(k, \theta^{*}\right)$-bounding does not produce any $D R$ but $(0.4, k)$-anonymity violates the privacy requirement more often due to overlooking duplicate records.

In summary, the performance of our $\operatorname{MS}\left(k, \theta^{*}\right)$-bounding is better than the other models, while Multi-sensitive $l$-diversity yields the worst performance.

\section{Results for frequency-based confidence setting}

Finally, we consider another scenario of confidence setting: the threshold of a symptom is set according to its frequency in the dataset. We calculated the frequencies of all symptoms appear in the dataset and set the confidence thresholds of the most $10 \%$ frequent symptoms to 1 , the last $10 \%$ frequent symptoms to 0.2 , and the remaining to 0.4 , respectively.
Again, we compared our MS( $\left.k, \theta^{\prime \prime}\right)$-bounding with Multisensitive $l$-diversity and $(\alpha, k)$-anonymity with the same parameter settings, i.e., $\alpha=0.2$ and 0.4 , and $k($ or $l)=5,10,15$, and 20. Figures 9 and 10 show the resulting NILs and DRs, respectively. From the obtained results, we observe that

1. As mentioned previously, all NILs generated except by $\operatorname{MS}\left(k, \theta^{\prime \prime}\right)$-bounding are the same as the uniform setting, while $D R$ s are different because of different threshold settings.

2. All models generate less $D R$ s than that by level-wise setting, because most dangerous groups appearing in the previous experiments are caused by high frequent symptoms, whose thresholds are set to 1 in this experiment.

3. Even $90 \%$ of $\theta^{\prime}$ s in $\theta^{\prime \prime}$ are set to 0.4 or lower, our $\operatorname{MS}\left(k, \theta^{\prime \prime}\right)$-bounding produces very small NIL than $(0.4, k)$-anonymity when being applied to data with high frequent symptoms such as 2004Q1 and 2007Q1.

In FAERS data, there are more than 20,000 different symptoms, which will require much researching effort and background knowledge to determine the threshold of each symptom. The frequency-based approach is a simple but reasonable method, and with this threshold definition, our $\operatorname{MS}\left(k, \theta^{\prime \prime}\right)$-bounding exhibits the best data utility and the least privacy risk among all the models we examined. 


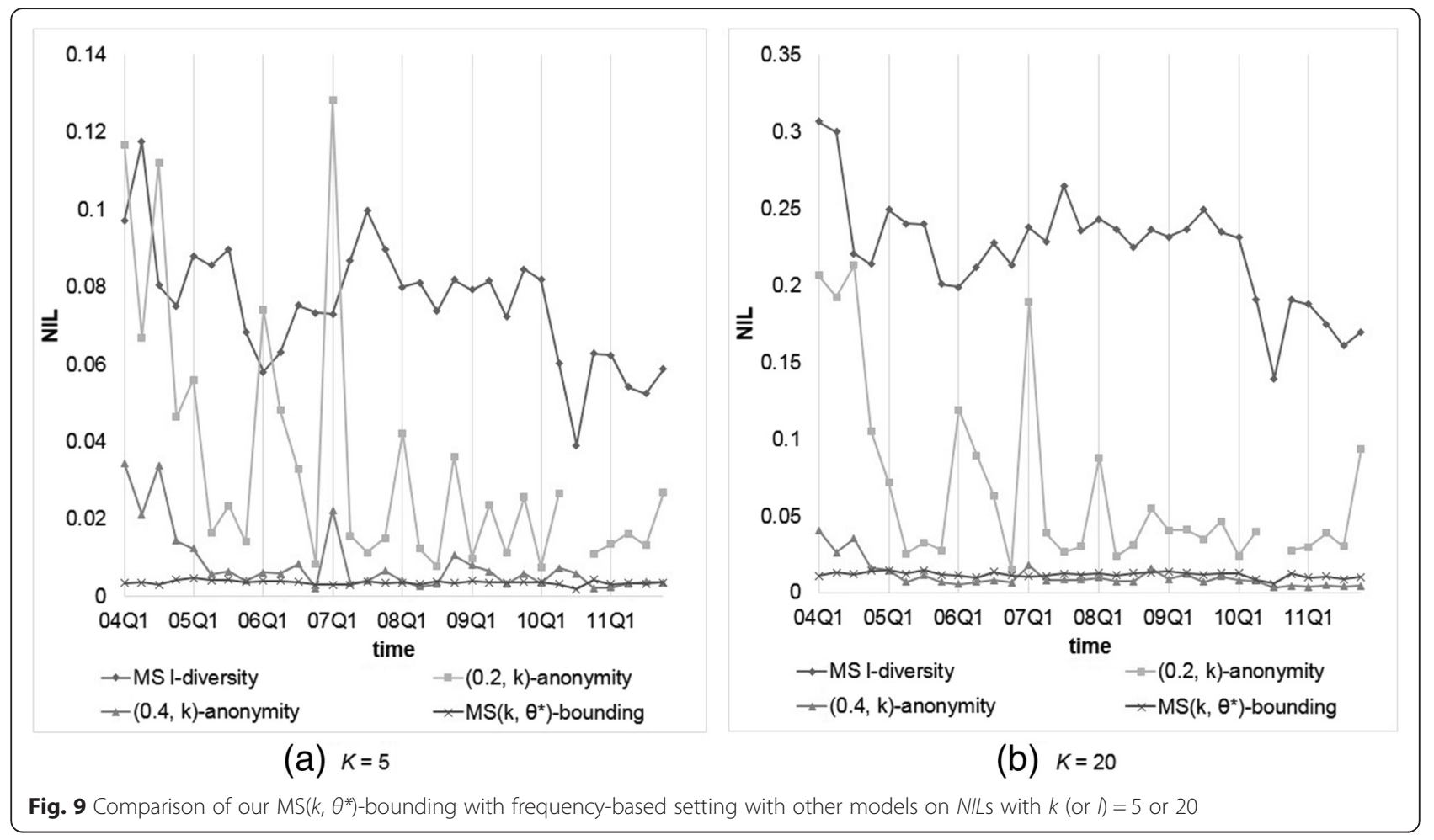

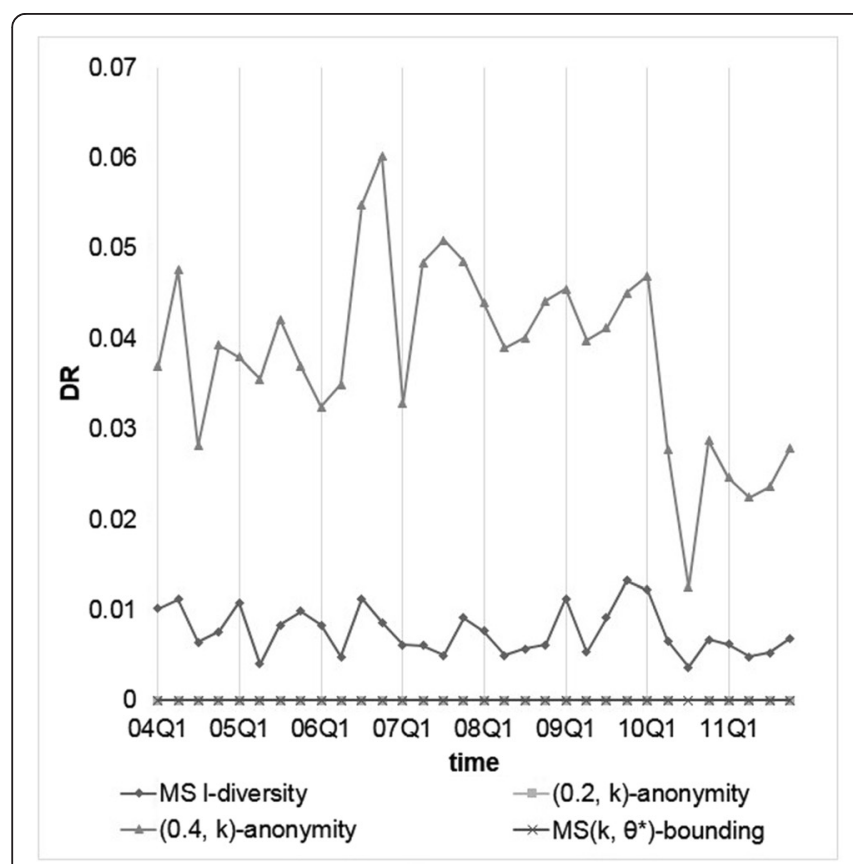

(a) $K=5$

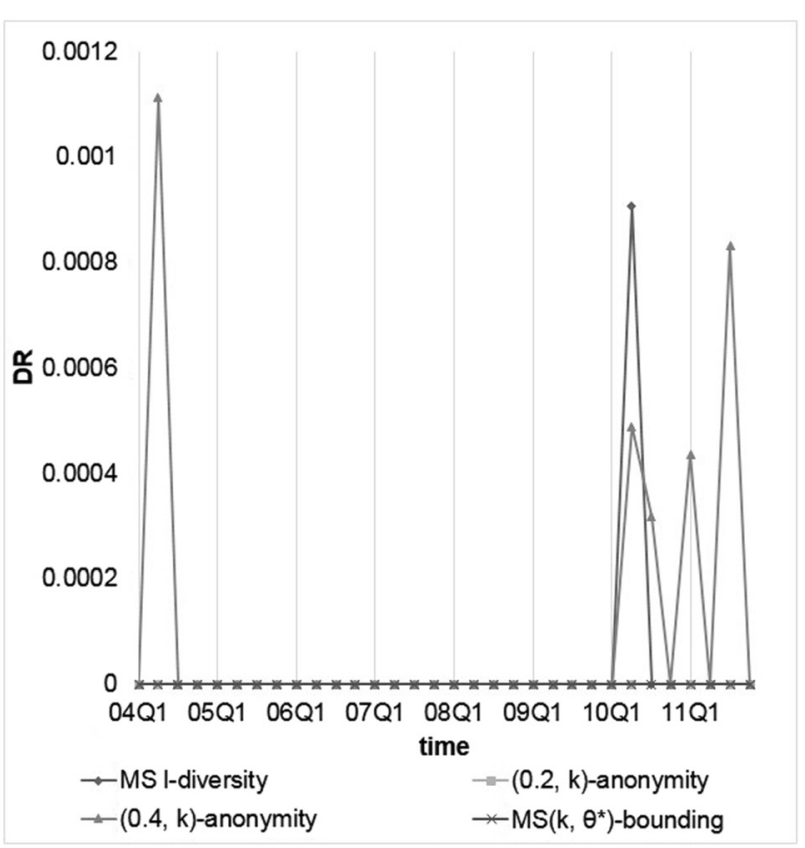

(b) $K=20$

Fig. 10 Comparison of our MS( $\left.k, \theta^{*}\right)$-bounding with frequency-based setting with other models on DRs with $k$ (or $\left.I\right)=5$ or 20 


\section{Influence on ADR signals}

We also conducted an experiment to inspect the impact of anonymized data on the strengths of discovered ADR rules. For each ADR rule shown in Table 5, we computed and checked the difference on the number of events, PRR and ROR measures between the original datasets and anonymized datasets. Since all rules exhibit similar phenomenon, we only show the results of the following rule

AVANDIA, age $>18 \Rightarrow$ CEREBROVASCULAR ACCIDENT.

Figure 11 depicts the occurrence and strength of the above rule in the original dataset (original count and original PRR) and from which the difference yielded from the dataset anonymized by Multi-sensitive $l$-diversity, $(0.2, k)$-anonymity, $(0.4, k)$-anonymity, and our $\operatorname{MS}\left(k, \theta^{*}\right)$ bounding with frequency-based setting, with $k=20$. The obtained results show that

1. Multi-sensitive $l$-diversity does not perform well because of the top-down strategy, which is less flexible to create QID-groups.

2. Most of the time there is no difference between the original and anonymized datasets except Multi-sensitive $l$-diversity. All of them are less than five, and the extreme value only occurs when original count is large (more than 80).

3. Not surprisingly, only very small difference on PRR ranging from -1 to 1 were observed from the anonymized datasets (except Multi-sensitive $l$-diversity), which nearly can be ignored.
These observations reveal that our method can effectively solve the privacy problem in SRS datasets without overlooking rare events and influencing the ADR signal strength.

\section{Conclusions}

In this paper, we proposed a new PPDP model for protecting SRS data that possess some characteristics overlooked by contemporary models, including rare events, multiple individual records, and multi-valued sensitive attributes. We also presented an anonymization algorithm to sanitize SRS data in accordance with the proposed model. Empirical studies showed that our method can prevent the disclosure of personal sensitive information without sacrificing the data utility and biasing the discovered ADR signals.

Although our approach is designed mainly for SRS data, it can also be applied to other types of medical data or applications with features analogous to SRS data; for example, electronic health records (EHRs), which contain more detailed private information and so deserve further investigation.

We also notice that FAERS data contain lots of missing values. Existing PPDP methods usually ignore the presence of missing values, simply deleting them before executing data anonymization. However, for data with enormous missing values, like SRS data, deleting all records with missing values may ruin the data utility seriously, so how to deal with missing values is an interesting

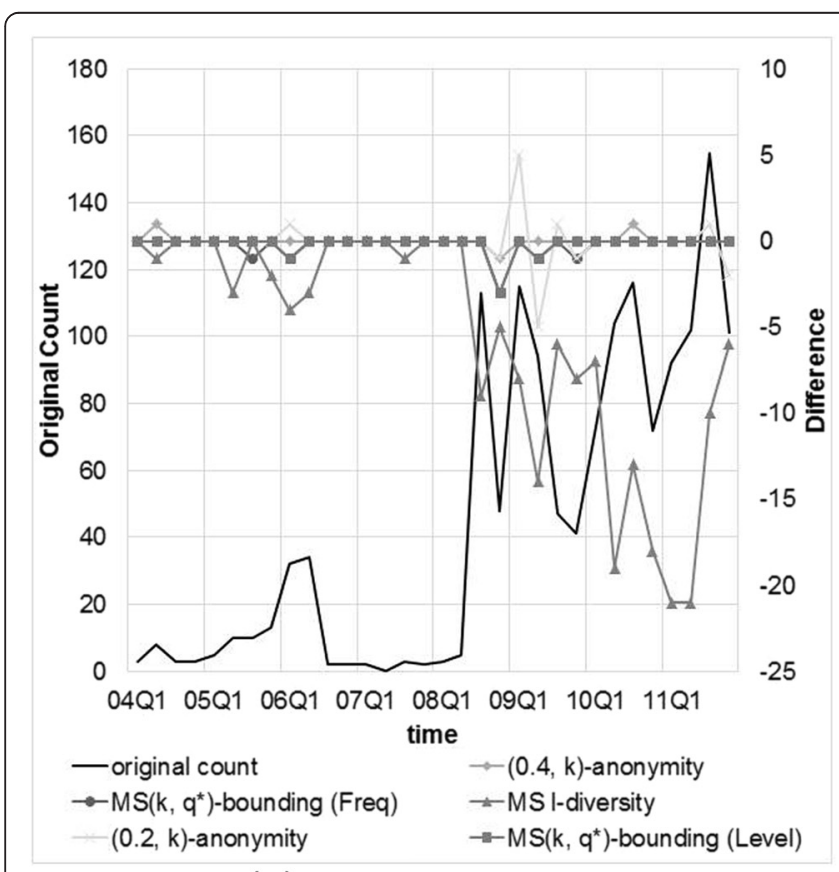

(a) Rule occurrence and difference

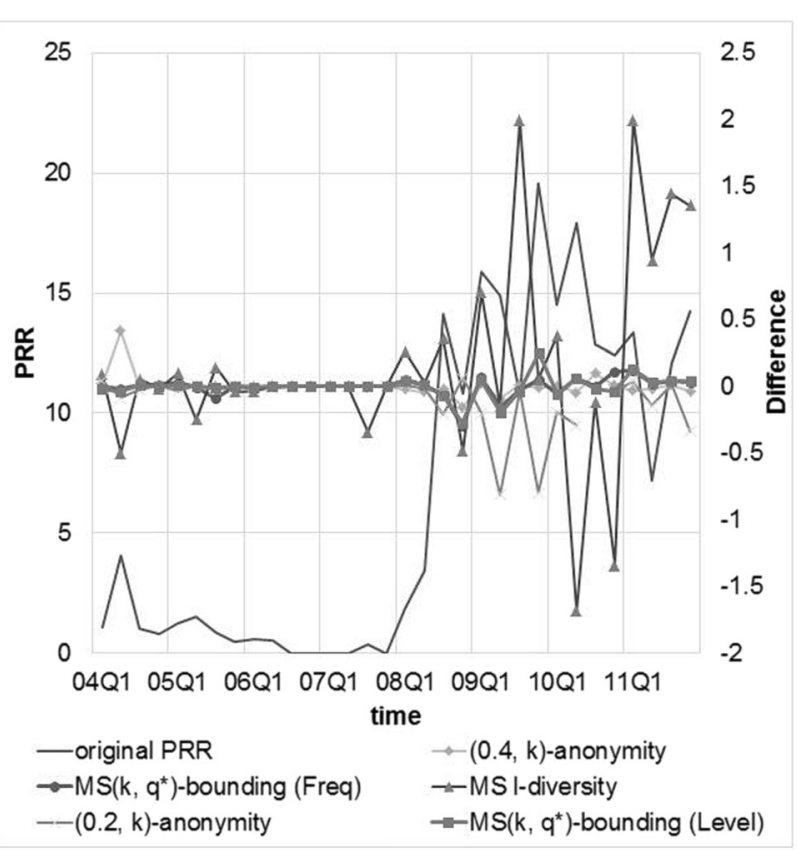

(b) Rule strength and difference

Fig. 11 Comparison of rule occurrence and strength (in PRR) generated from original dataset and anonymized dataset by different methods 
issue. Another important and challenging issue goes to continuous data publishing $[11,19]$. Typically, SRS data are released sequentially. Combining related releases would sharpen the identification of an individual record or sensitive information. We are endeavoring to extend our current approach to solve these problems.

\section{Acknowledgements}

This problem was initiated when WYL was on sabbatical leave to University of Maryland, Baltimore County in 2011. He especially appreciates Prof. Hillol Kargupta for his gracious hospitality and valuable discussion on this topic. This work was supported in part by the Ministry of Science and Technology of Taiwan under grant No. MOST102-2221-E-390-020.

\section{Availability of data and materials}

FDA Adverse Event Reporting System (FAERS): Latest Quarterly Data Files. http://www.fda.gov/Drugs/GuidanceComplianceRegulatorylnformation/ Surveillance/AdverseDrugEffects/ucm082193.htm Accessed 2 Jan 2016.

\section{Authors' contributions}

WYL and DCY worked in collaboration in every aspect of this research, while DCY was responsible for the implementation of the algorithm. JTW helped to carry out the experiments and the revision of this paper. All authors read and approved the final manuscript.

\section{Competing interests}

The authors declare that they have no competing interests. This research received no specific grant from any funding agency in the public, commercial, or not-for-profit sectors.

\section{Consent for publication}

Not applicable.

Ethics of approval and consent to participate

Not applicable.

\section{Declarations}

Publication of this article was funded by the corresponding author. This article has been published as part of BMC Medical Informatics and Decision Making Volume 16 Supplement 1, 2016: Proceedings of the ACM Ninth International Workshop on Data and Text Mining in Biomedical Informatics. The full contents of the supplement are available online at https://bmcmedinformdecismak.biomedcentral.com/articles/supplements/ volume-16-supplement-1.

Published: 18 July 2016

\section{References}

1. FDA Adverse Event Reporting System (FAERS). http://www.fda.gov/cder/ aers/default.htm Accessed 2 Jan 2016.

2. The Yellow Card Scheme. https://yellowcard.mhra.gov.uk/ Accessed 2 Jan 2016.

3. MedEffect Canada. http://www.healthcanada.gc.ca/medeffect Accessed 2 Jan 2016.

4. Fung BCM, Wang K, Chen R, Yu PS. Privacy-preserving data publishing: A survey of recent developments. ACM Comput Surv. 2010;42(4):14.

5. Lin WY, Yang DC. On privacy-preserving publishing of spontaneous ADE reporting data. In: Proceedings of 2013 IEEE International Conference on Bioinformatics and Biomedicine. 2013. p. 51-3.

6. Gould AL. Practical pharmacovigilance analysis strategies. Pharmacoepidemiol Drug Saf. 2003;12(7):559-74.

7. Evans SJ, Waller PC, Davis S. Use of proportional reporting ratios (PRRs) for signal generation from spontaneous adverse drug reaction reports. Pharmacoepidemiol Drug Saf. 2001;10(6):483-6.

8. Egberts AC, Meyboom RH, van Puijenbroek EP. Use of measures of disproportionality in pharmacovigilance: three dutch examples. Drug Saf. 2002;25(6):453-8.

9. Sweeney L. k-anonymity: A model for protecting privacy. Int J Uncertainty Fuzziness Knowledge Based Syst. 2002;10(5):557-70.

10. Machanavaijhala A, Gehrke J, Kifer D, Venkitasubramaniam M. I-diversity: Privacy beyond k-anonymity. ACM Trans Knowl Discov Data. 2007;1(1):3.
11. Wang K, Fung BCM. Anonymizing sequential releases. In: Proceedings of 12th ACM SIGKDD International Conference on Knowledge Discovery and Data Mining. 2006. p. 414-23.

12. Shi $P$, Xiong $L$, Fung BCM. Anonymizing data with quasi-sensitive attribute values. In: Proceedings of 19th ACM International Conference on Information and Knowledge Management. 2010. p. 1389-92.

13. Wong RCW, Li J, Fu AWC, Wang K. (a, k)-anonymity: An enhanced kanonymity model for privacy preserving data publishing. In: Proceedings of 12th ACM SIGKDD International Conference on Knowledge Discovery and Data Mining. 2006. p. 754-9.

14. Gal TS, Chen Z, Gangopadhyay A. A privacy protection model for patient data with multiple sensitive attributes. Int J Inf Secur Priv. 2008;2(3):28-44.

15. Byun JW, Kamra A, Bertino E, Li N. Efficient k-anonymization using clustering techniques. In: Proceedings of the 12th International Conference on Database Systems for Advanced Applications. 2007. p. 188-200.

16. MeSH (Medical Subject Headings) Tree Structures. http://www.nlm.nih.gov/ mesh/MBrowser.html Accessed 2 Jan 2016.

17. Lin WY, Lan L, Huang FS. Rough-set-based ADR signaling from spontaneous reporting data with missing values. J Biomed Inform. 2015;58:235-46.

18. MedWatch. http://www.fda.gov/Safety/MedWatch Accessed 2 Jan 2016.

19. Fung BCM, Wang K, Fu AWC, Pei J. Anonymity for continuous data publishing. In: Proceedings 11th International Conference on Extending Database Technology: Advances in Database Technology. 2008. p. 264-75.
Submit your next manuscript to BioMed Central and we will help you at every step:

- We accept pre-submission inquiries

- Our selector tool helps you to find the most relevant journal

- We provide round the clock customer support

- Convenient online submission

- Thorough peer review

- Inclusion in PubMed and all major indexing services

- Maximum visibility for your research

Submit your manuscript at www.biomedcentral.com/submit
) Biomed Central 